\title{
Dynamic distribution of muscle-specific calpain in mice has a key role in physical-stress adaptation and is impaired in muscular dystrophy
}

\author{
Koichi Ojima,1,2,3 Yukiko Kawabata,2,4,5 Harumi Nakao,5,6 Kazuki Nakao,7 Naoko Doi,1,2 \\ Fujiko Kitamura, ${ }^{1}$ Yasuko Ono, ${ }^{1,2}$ Shoji Hata, ${ }^{1}$ Hidenori Suzuki, ${ }^{8}$ Hiroyuki Kawahara, ${ }^{9}$ \\ Julius Bogomolovas, ${ }^{10}$ Christian Witt, ${ }^{10}$ Coen Ottenheijm, ${ }^{11}$ Siegfried Labeit, ${ }^{10}$ Henk Granzier, ${ }^{11}$ \\ Noriko Toyama-Sorimachi, ${ }^{12}$ Michiko Sorimachi, ${ }^{13}$ Koichi Suzuki, ${ }^{14,15}$ Tatsuya Maeda, ${ }^{2,15}$
} Keiko Abe, ${ }^{4}$ Atsu Aiba, $2,5,6$ and Hiroyuki Sorimachi1,2,4,15

${ }^{1}$ Calpain Project, The Tokyo Metropolitan Institute of Medical Science (Rinshoken), Tokyo, Japan. ${ }^{2}$ CREST, JST, Kawaguchi, Japan. ${ }^{3}$ Meat Proteins Research Team, National Institute of Livestock and Grassland Science (NILGS), Tsukuba, Japan. ${ }^{4}$ Graduate School of Agricultural and Life Sciences, The University of Tokyo, Tokyo, Japan. ${ }^{5}$ Division of Molecular Genetics, Kobe University Graduate School of Medicine, Kobe, Japan. ${ }^{6}$ Graduate School of Medicine, The University of Tokyo, Tokyo, Japan. 7Laboratory for Animal Resources and Genetic Engineering RIKEN, Center for Developmental Biology, Kobe, Japan.

${ }^{8}$ Laboratory for Electron Micrography, The Tokyo Metropolitan Institute of Medical Science (Rinshoken), Tokyo, Japan. ${ }^{9}$ Department of Biological Sciences,

Tokyo Metropolitan University, Hachioji, Japan. ${ }^{10}$ Institute for Integrative Pathophysiology, Universitätsklinikum Mannheim, Mannheim, Germany.

${ }^{11}$ Department of Molecular and Cellular Biology, University of Arizona, Tucson, Arizona, USA. ${ }^{2}$ Department of Gastroenterology, Research Institute, National Center for Global Health and Medicine, Tokyo, Japan. ${ }^{13}$ The Institute of Research and Development, Polytechnic University, Sagamihara, Japan.

${ }^{14}$ New Frontiers Research Laboratory, Toray Industries Inc., Kamakura, Japan.

${ }^{15}$ Institute of Molecular and Cellular Biosciences, The University of Tokyo, Tokyo, Japan.

\begin{abstract}
Limb-girdle muscular dystrophy type 2A (LGMD2A) is a genetic disease that is caused by mutations in the calpain 3 gene (CAPN3), which encodes the skeletal muscle-specific calpain, calpain 3 (also known as p94). However, the precise mechanism by which $\mathrm{p} 94$ functions in the pathogenesis of this disease remains unclear. Here, using p94 knockin mice (termed herein P94KI mice) in which endogenous p94 was replaced with a proteolytically inactive but structurally intact p94:C129S mutant protein, we have demonstrated that stretch-dependent p94 distribution in sarcomeres plays a crucial role in the pathogenesis of LGMD2A. The P94KI mice developed a progressive muscular dystrophy, which was exacerbated by exercise. The exercise-induced muscle degeneration in $\mathrm{p} 94 \mathrm{KI}$ mice was associated with an inefficient redistribution of $\mathrm{p} 94: \mathrm{C} 129 \mathrm{~S}$ in stretched sarcomeres. Furthermore, the $\mathrm{p} 94 \mathrm{KI}$ mice showed impaired adaptation to physical stress, which was accompanied by compromised upregulation of muscle ankyrin-repeat protein- 2 and hsp upon exercise. These findings indicate that the stretchinduced dynamic redistribution of $\mathrm{p} 94$ is dependent on its protease activity and essential to protect muscle from degeneration, particularly under conditions of physical stress. Furthermore, our data provide direct evidence that loss of P94 protease activity can result in LGMD2A and molecular insight into how this could occur.
\end{abstract}

\section{Introduction}

Limb-girdle muscular dystrophy (LGMD) is a genetically heterogeneous group of disorders involving progressive muscle weakness and atrophy of the truncal and proximal limb muscles, elevated serum creatine kinase (CK) levels, and dystrophic changes visible in muscle biopsy sections (1-4). To date, 452 distinct pathogenic mutations of the CAPN3 gene, which encodes p94/calpain 3 , have been found in LGMD2A patients $(5,6)$. p94 is a member of the calpain family, which consists of $\mathrm{Ca}^{2+}$-requiring intracellular cysteine proteases that cleave their substrates at a few specific sites to modulate the substrate's function $(7,8)$. The mutations of the p94-encoding CAPN3 gene are distributed along the entire length of the gene product and include missense, nonsense, frameshift, and deletion mutations (3, 6, 9-11). These findings, as well

Authorship note: Koichi Ojima and Yukiko Kawabata contributed equally to this work.

Conflict of interest: The authors have declared that no conflict of interest exists. Citation for this article: J Clin Invest. 2010;120(8):2672-2683. doi:10.1172/JCI40658 as p94 $\mathrm{Tg}(12)$ and $\mathrm{KO}$ mouse studies $(13,14)$, indicate that p94 plays an essential role(s) in skeletal muscle. However, the precise mechanism by which the diverse p94 mutations contribute to the LGMD2A pathology is still largely unknown.

In myofibrils, p94 directly interacts with connectin/titin at the M-line and N2A regions (15), where multiple protein complexes that use connectin/titin as a scaffold form. These complexes are thought to associate with myofibrillar signal-transduction systems (16). In the N2A region of connectin/titin, a binding site for muscle ankyrin repeat proteins (MARPs), which are stressresponse transcriptional regulator proteins, overlaps with that for p94, and MARP2/Ankrd2 is proteolyzed by p94 with the structural aid of connectin/titin, which may modulate stressdependent signal transduction $(17,18)$. Our previous in vitro study demonstrated that p94 shifts its location from the M-line to the $\mathrm{N} 2 \mathrm{~A}$ region when the sarcomere is extended, suggesting that p94 functions as a sarcomere-length sensor in cooperation with connectin/titin (19) to mediate signal transduction in response to external stress. 

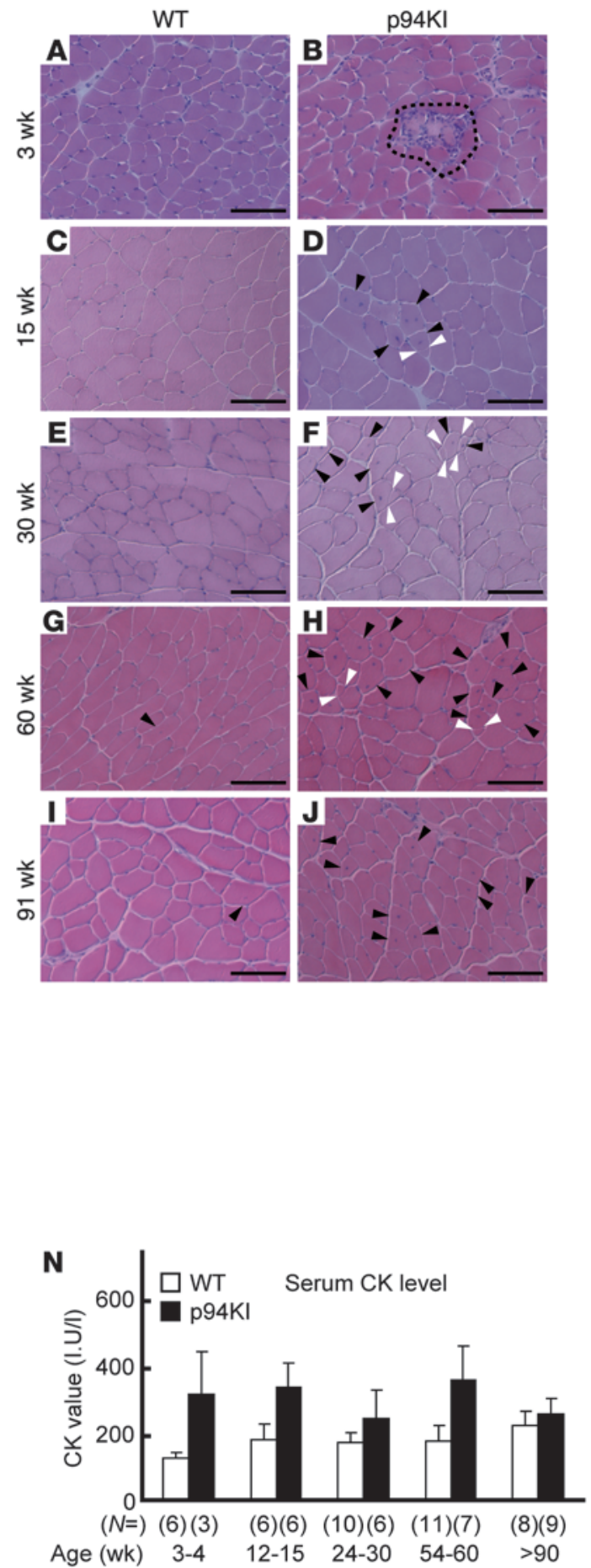
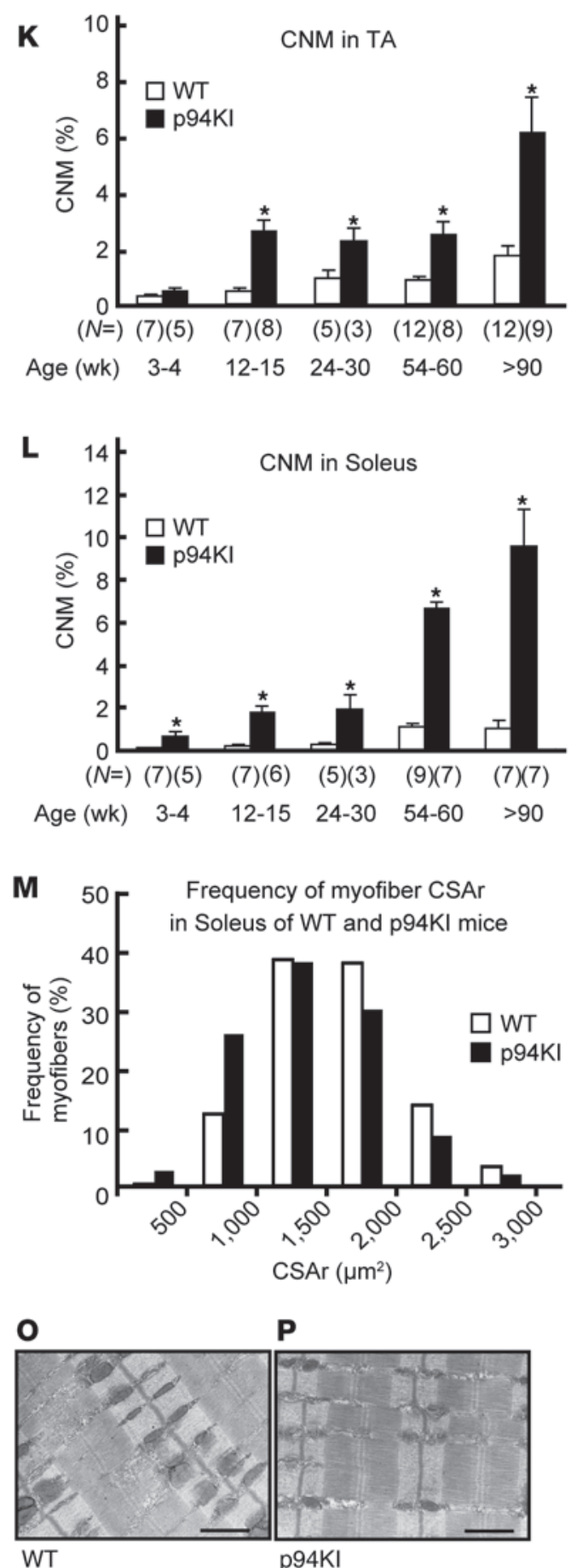

Figure 1

MD phenotypes in p94KI mice. (A-J) H\&E-stained TA muscle sections from WT (A, C, E, G, and I) and p94KI (B, D, F, H, and J) mice of the ages indicated. p94KI showed typical dystrophic phenotypes, such as cell invasion (dotted circles), central nuclei (black arrowheads), and the splitting of myofibers (white arrowheads). Scale bars: $100 \mu \mathrm{m}$. (K and $\mathbf{L}$ ) Increased CNM during aging in p94KI mice. The number of CNM in the TA (K) and soleus (L) was compared with the number of total myofibers. (M) Frequency distribution of myofiber CSArs in the soleus of mice over 90 weeks old. A total of 302 fiber profiles was traced in each section from 3 mice of each type. The average myofiber CSAr was $1,542 \mu m^{2}$ in WT mice and 1,342 $\mu \mathrm{m}^{2}$ in p94KI mice. The fiber size in p94KI mice was significantly smaller than in WT mice $(P<0.01)$. White bars, WT; black bars, p94KI. (N) The mean serum CK level (IU/I) in p94KI mice appeared higher than in WT, but this difference was not statistically significant. ${ }^{*} P<0.05$ versus WT. ( $\mathbf{O}$ and $\mathbf{P}$ ) Ultrastructural analysis showed a typical striated pattern with properly assembled sarcomeres in a 91 -week-old p94KI mouse (P) with no apparent difference from the pattern of a 26-week-old WT mouse (0). Scale bars: $1 \mu \mathrm{m}$. 
Here, we hypothesized that the p94 protease activity is essential for the signal-transduction pathway by which muscles adapt to physical stress, the perturbation of which leads to LGMD2A. To test this hypothesis, we generated p94:C129S knockin (p94KI) mice that express a protease-inactive p94 mutant in place of WT p94. The p94KI mice, in contrast to conventional KO (p94KO) mice, express a $\mathrm{p} 94: \mathrm{C} 129 \mathrm{~S}$ protein with an intact structure under the control of the endogenous p94 promoter. The p94KI mice showed MD phenotypes, which were exacerbated by exercise. Importantly, our results showed that the p 94 protease activity contributed to the dynamic change in the distribution of p94 in myocytes in response to sarcomere conditions and its loss impaired a stress-response pathway involving MARPs and hsp. Thus, our p94KI mouse enabled us to assess the loss of p94 protease function in skeletal muscle without superimposing potential structural defects and provided evidence linking the dysfunction of p94 with the LGMD2A pathological mechanism.

\section{Results}

p94KI mice showed progressive muscle degeneration/regeneration during aging. To address the role of $\mathrm{p} 94$ in LGMD2A pathogenesis, we created mice that expressed p94:C129S in place of WT p94 (Supplemental Figure 1; supplemental material available online with this article; doi:10.1172/JCI40658DS1). The resultant p94KI mice were fertile, with a normal lifespan. However, the skeletal muscles (tibialis anterior [TA] and soleus) of the p94KI mice showed typical MD symptoms, such as split myofibers and centrally located nuclei, and this pathology was exacerbated with age (Figure 1, A-J). The proportion of centrally nucleated myofibers (CNM) in both the TA and soleus of p94KI mice was significantly higher than in WT mice (Figure 1, K and L). The average myofiber cross-sectional areas (CSArs) in the soleus muscles of WT and p94KI mice at more than 90 weeks of age were $1,542 \mu \mathrm{m}^{2}$ and $1,342 \mu \mathrm{m}^{2}$, respectively, which were significantly different $(P<0.01)$ (Figure $1 \mathrm{M})$. The serum CK level, another typical marker for MD indicative of muscle membrane damage, was not significantly different between the p94KI and WT mice, demonstrating that no significant membrane damage occurred in the p94KI mice (Figure 1N). The ultrastructure of the p94KI mouse muscle was also normal and not markedly different from that of WT mice (Figure 1, O and P). Thus, the p94KI mice had a gradually progressive MD phenotype that worsened with age without severe sarcolemmal disruption. In contrast, both human patients with CAPN3-null mutations and $\mathrm{p} 94 \mathrm{KO}$ mice show a more severe MD phenotype $(9,10,13,14)$. This difference in disease severity between these cases of MD and that seen in the p94KI mice suggests that $\mathrm{p} 94$ has functions other than its role in proteolysis that are involved in MD pathology.

Exercise-provoked muscle degeneration in p94KI mice. In humans, eccentric exercise has been shown to cause p94 autolysis (20). This observation suggests that the $\mathrm{p} 94$ protease activity plays a role in the adaptive response of muscles to exercise. Therefore, we next investigated whether exercise affects MD pathogenesis in $\mathrm{p} 94 \mathrm{KI}$ mice. We subjected p94KI and WT mice to downhill treadmill running and assessed the biochemical and pathological changes in their skeletal muscles. Immunoblot analysis revealed that p94 underwent proteolysis during exercise (Figure 2, A and B) and that its distribution shifted from the precipitate (Ppt; myofibril) to the supernatant (Sup; cytosol) fraction in WT but not in p94KI mice during exercise (Supplemental Figure 2). The degeneration of myo- fibers due to membrane disruption, as revealed by the incorporation of Evans blue dye (EBD), was clearly observed in the exercised p94KI (p94KI-ex) mice but was barely detectable in the exercised WT (WT-ex) mice (Figure 2, C-N). EBD-containing myofibers were observed among all the CSAr classes in the p94KI-ex muscle, indicating that membrane disruption was not exacerbated in particular classes of muscle fibers (Supplemental Figure 3). Consistent with these findings, the serum CK level in the p94KI-ex mice was significantly higher than in the WT-ex or unexercised p94KI mice (Figure 2O). The proportion of CNMs was also significantly increased in the $\mathrm{p} 94 \mathrm{KI}$ but not WT mice 10 days after exercise (Figure 2, $\mathrm{P}$ and Q). These results clearly indicate that the $\mathrm{p} 94$ protease activity is required to protect myofibers from undergoing degeneration under exercise-induced stress.

Skeletal muscles have the ability to self repair rapidly in response to injury or disease (21). After exercise, the damaged muscles in the p94KI mice began to regenerate (Supplemental Figure 4). The infiltration of mononuclear cells into the injured muscle was observed on postexercise days $1-3$, and regenerating thin myofibers were observed on postexercise day 10, although these myofibers were heterogeneous, with the appearance of dystrophic degeneration. These results suggested that a lack of $\mathrm{p} 94$ proteolytic activity does not affect the regenerative potential of satellite cells.

The $p 94$ exchange rate in myofibrils is fine tuned by its protease activity. To understand the molecular mechanisms by which p 94 protects muscles, we analyzed the intracellular behavior of p94. We previously demonstrated that p94 has a stretch-dependent distribution (19) and that it associates physically and functionally with sarcomeric proteins, including connectin/titin and MARP2 (17). We therefore compared the dynamic behavior of p94 in WT and p94KI mouse myocytes using fluorescence recovery after photobleaching (FRAP) with GFP-tagged WT p94 and p94:C129S (GFP-p94WT and GFP-p94CS) expressed in primary cultured myotubes from WT and p94KI mice. The GFP signals for both GFP-p94WT and GFP-p94CS were predominantly detected at the M-line of connectin/titin (ref. 19 and Figure 3, A and B). The FRAP experiments revealed that the GFP-p94WT proteins at the M-line were dynamically mobile, with a recovery $t_{1 / 2}$ of $66.5 \mathrm{~s}$ (Figure $3 \mathrm{C}$ ). This turnover rate was nearly as rapid as that of the $\alpha$-actinin fast mobile fraction $\left(t_{1 / 2}=61 \pm 11 \mathrm{~s}\right)(22)$. Notably, GFP-p94CS showed a significantly longer recovery $t_{1 / 2}(129 \mathrm{~s})$ than GFP-p94WT (Figure 3C; Supplemental Figures 5 and 6). These results indicated that p94 is dynamically located in the strictly organized sarcomere and that its mobility rate is fine tuned by its protease activity.

p94's protease activity is required for its dynamic redistribution in response to mechanical loading. Many myofibril-associated proteins that display a dynamic distribution within myofibers are mechanically sensitive and are thought to play an important role in the adaptive response to mechanical perturbation (23). Therefore, we further investigated the dynamic behavior of p94 in response to mechanical loading. We previously reported that the myofibrillar localization of exogenously expressed p94 shifts from the M-line to the N2A region of connectin/titin during sarcomeric stretch, using cultured myocytes (19). Consistent with this result, we found that p94 was predominantly located at the N2A region of stretched sarcomeres in both WT and p94KI mice (Figure 3, D-O). Importantly, the $\mathrm{M} / \mathrm{N} 2 \mathrm{~A}$ ratio of $\mathrm{p} 94$ became smaller as the sarcomeres stretched (Figure 3P). This means that as the sarcomeres extend, p94 tends to accumulate in the N2A region. These results 
A

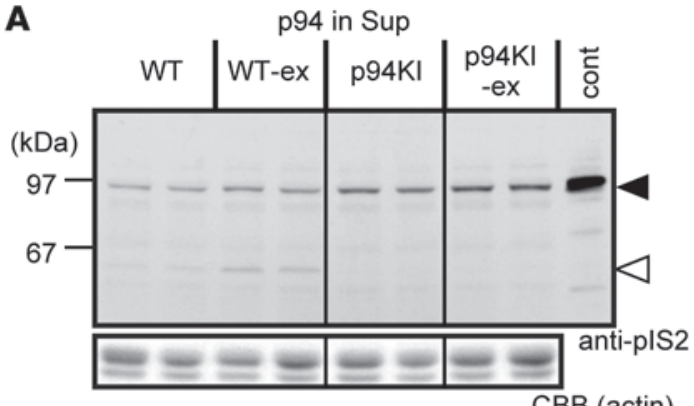

B

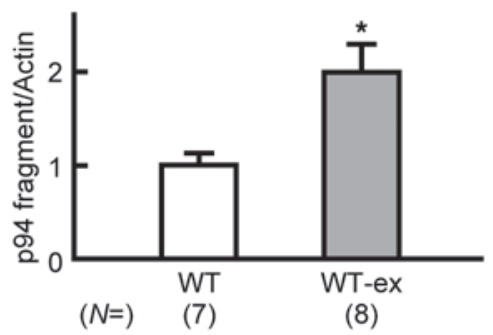

$(N=)$ p94 fragment in Sup

(7)
(8)

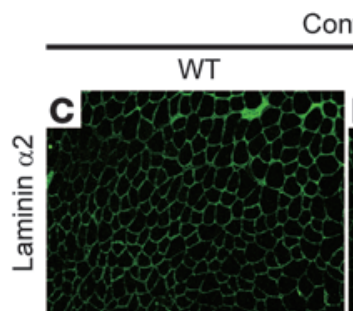

Control

Postexercise
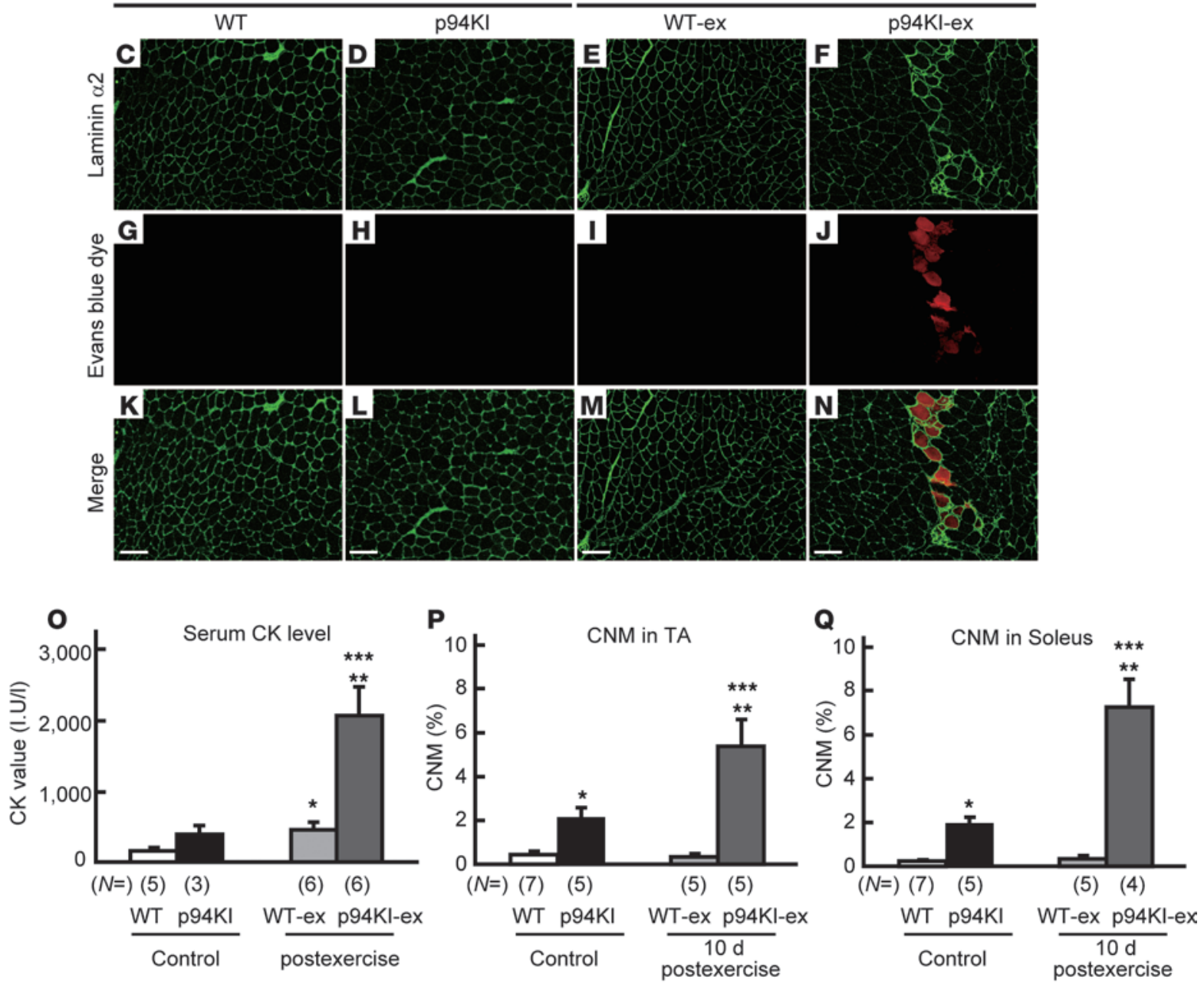

Figure 2

Exercise-induced myofiber degeneration in p94KI. (A and B) Immunodetection of p94 and its fragments (black and white arrowheads, respectively) in WT, WT-ex, p94KI, and p94KI-ex mice. The Sup fraction from TA was subjected to Western blot analysis using an anti-plS2C Ab (A). Hairlines indicate lanes that were run on the same gel but were noncontiguous. The p94 bands were quantified by normalizing to the CBB-stained actin (CBB in A) (B). cont, COS-expressed p94:C129S. ${ }^{*} P<0.01$. (C-N) Damaged myofibers in the gastrocnemius muscles from WT, WT-ex, p94KI, and p94KI-ex mice were detected by an anti-laminin $\alpha 2 \mathrm{Ab}(\mathbf{C}-\mathbf{F})$ and EBD autofluorescence (G-J). Merged images plus nuclei (K-N). Scale bars: $100 \mu \mathrm{m}$. (O) Drastic increase in the serum CK level in p94KI-ex. Blood samples of WT-ex and p94KI-ex were taken immediately after excise. ( $\mathbf{P}$ and $\mathbf{Q})$ The proportion of CNM in p94KI was significantly increased after exercise. The TA (P) and soleus (Q) from WT, p94KI, WT-ex, and p94KI-ex sacrificed 10 days after exercise were analyzed. The proportion of CNMs was calculated as in Figure $1 .{ }^{*} P<0.05$ versus WT; ${ }^{*} P<0.05$ versus $\mathrm{p} 94 \mathrm{KI} ;{ }^{* * *} P<0.05$ versus WT-ex. 

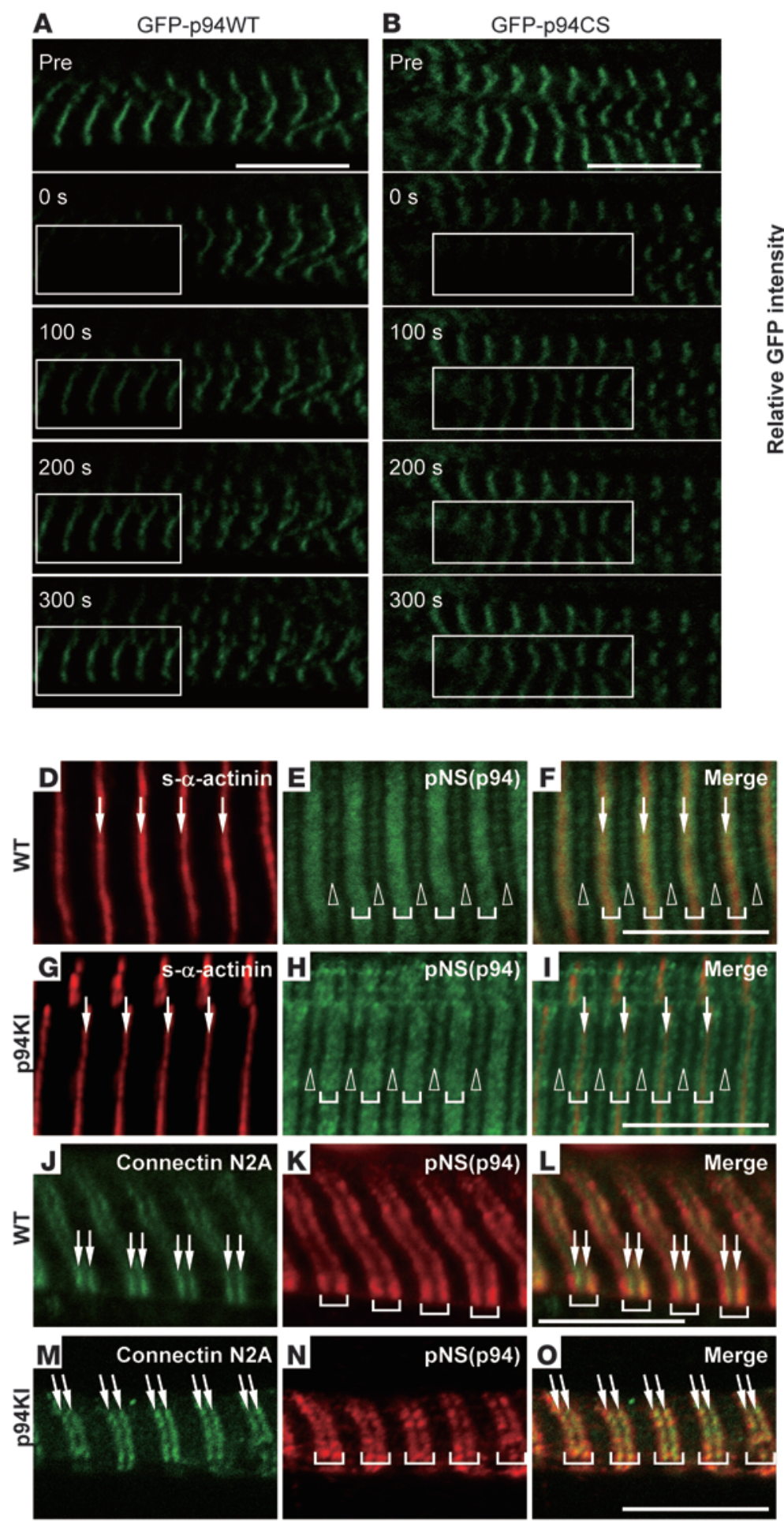
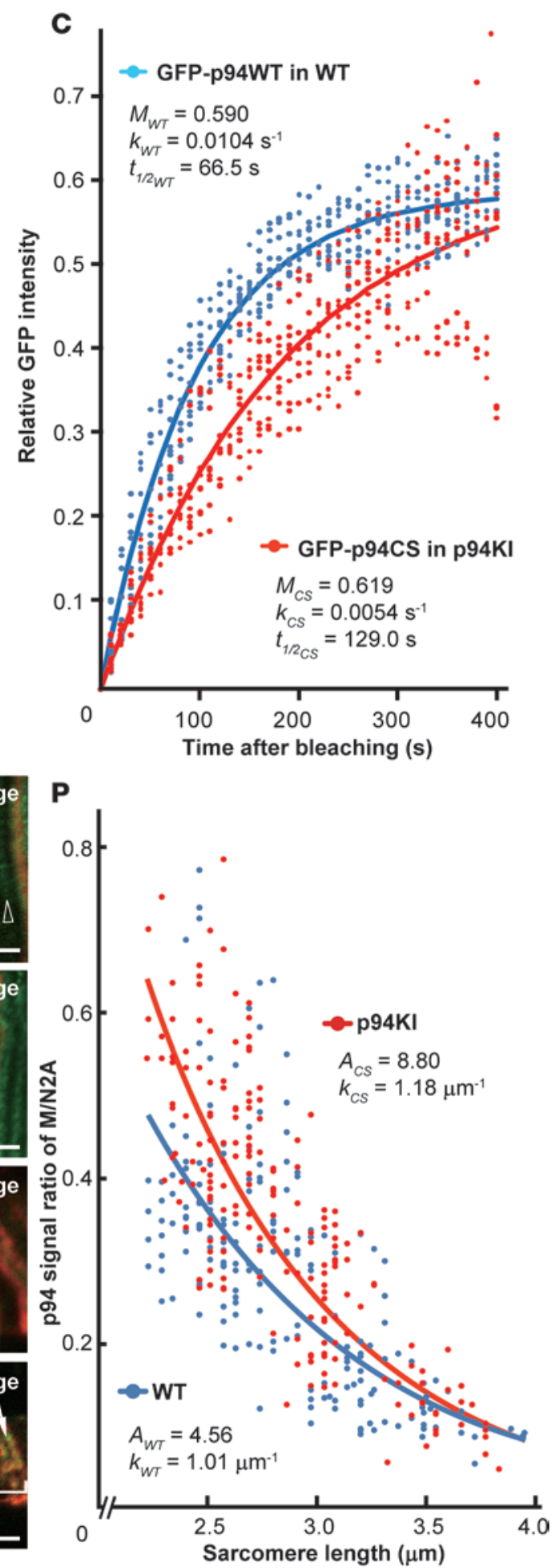


\section{Figure 3}

Translocation of p94 in response to sarcomeric length. (A-C) Direct observation of rapid GFP-p94 exchange in cultured myotubes by FRAP. GFP-p94WT and GFP-p94CS were exogenously expressed in cultured myotubes from WT (A) and p94KI (B) mice and were predominantly localized to the M-lines of connectin/titin. Reference images were acquired before photobleaching (Pre). Rectangles indicate the photobleached areas. The signals in the M-line were calculated as the ratio of GFP intensity at the indicated time points, and exponential curves were fitted $(\mathbf{C})$. The mobile fraction $(\mathrm{M})$, rate constant $(k)$, and recovery half-time $\left(t_{1 / 2}\right)$ were calculated from the FRAP curve. Note that a single exponential fit provided the best result, and $k$ (i.e., $t_{1 / 2}$ ) was significantly different between WT and p94KI (see Supplemental Figures 5 and 6). (D-P) Confocal micrographs of p94 localization in longitudinal sections of EDL from WT (D-F and $\mathbf{J}-\mathbf{L}$ ) and p94KI (G-I and $\mathbf{M}-\mathbf{O}$ ) mice. p94, the Z-bands, and connectin/titin N2A region were detected with anti-pNS (E, H, K, and $\mathbf{N}$ ), anti-s- $\alpha$-actinin (D and G; arrows), and anti-connectin/titin-N2A ( $\mathbf{J}$ and $\mathbf{M}$; arrows) Abs, respectively. p94 was localized to the M-lines (open arrowheads), N2A regions, and/or Z-bands (brackets) of both mice, but predominantly to the N2A regions in extended sarcomeres ( $\mathbf{K}$ and $\mathbf{N}$ ). Ratio of the p94 signal in the $\mathbf{M}$-line to that in the N2A region was plotted against the sarcomeric length $(\mathbf{P})$. The 2 estimated lines were statistically shown to be significantly different (see Supplemental Figure 6 legend). Scale bars: $10 \mu \mathrm{m}$.

indicate that the amount of $\mathrm{p} 94$ that dynamically redistributes from the M-line to the $\mathrm{N} 2 \mathrm{~A}$ region can quantitatively reflect the exercise load of myofibers. Importantly, in p94KI mice, the accumulation of p94 in the N2A region during sarcomeric extension took significantly longer than in WT, indicating that the p94 protease activity is required for p94's rapid dynamic redistribution in response to mechanical loading.

Loss of $p 94$ protease activity impairs the MARP2 response to physical stress. What is the significance of the p94 redistribution from the M-line to the N2A region in LGMD2A pathogenesis? Given the importance of the connectin/titin N2A region as a scaffold for various signaling molecules $(18,24)$, we hypothesized that the exercisedependent redistribution of p94 regulates signaling molecules at the $\mathrm{N} 2 \mathrm{~A}$ region, inducing an adaptive response to protect muscles from exercise-induced degeneration. To test this hypothesis, we focused on MARP2/ANKRD2, since MARP2 interacts directly with connectin/titin at the N2A region $(15,25)$, where it competes with p94 (17). In addition, MARP2's expression is sensitive to muscle conditions $(26,27)$, and it modulates transcription factors such as YB1, PML, and p53 by binding them (28). Consistent with these reports, we observed that the MARP2 protein was increased in WTex mice compared with WT mice (Figure 4, A and B; Supplemental Figure 7). In contrast, the p94KI-ex mice showed a significant decrease in MARP2 in comparison with all the other types of mice. This impairment in MARP2 induction upon exercise was also confirmed by immunohistochemical analysis (Figure 4, C-N; Supplemental Figure 8). MARP2-positive nuclei were found in WT, p94KI, WT-ex, and p94KI-ex mice as $2.3 \%, 1.3 \%, 14 \%$, and $4.8 \%$ of the total nuclei, respectively. These were confirmed to be myonuclei and did not originate from satellite cells (Supplemental Figure 9). These results suggested the important notion that the $\mathrm{p} 94$ protease activity is required for MARP2 induction in response to exercise.

Next, we determined whether the MARP2 induction upon exercise has a role in preventing muscle degeneration. WT and p94KI mice, into which EBD was injected before exercise, were subjected to downhill running on a treadmill and sacrificed immediately after the exercise. In the WT-ex mice, EBD was incorporated into several myofibers in which MARP2 expression was induced, although not all the MARP2-positive myofibers were positive for EBD (Figure 5, A-C). Even in the EBD-positive myofibers, the localization of dysferlin was normal (Figure 5, G-I), indicating that the sarcolemma was not markedly disrupted, as previously reported $(29,30)$. In contrast to the WT-ex mice, in the p94KI-ex mice, the myofibers that were positive for EBD showed weaker MARP2 signals and abnormal dysferlin localization (Figure 5, D-F and J-L). These results indicated that the exercise provoked plasma membrane disruption and myofiber degeneration in the p94KI mice.

Molecular states of $p$ 94KI mice mimic those of WT-ex mice. To further examine pathophysiological alterations in p94KI mice, we analyzed their proteomes using TA muscles from WT, WT-ex, p94KI, or p94KI-ex mice with the iTRAQ/LC-MALDI systems. Among 660 proteins identified, 44 were upregulated (p94KI/WT > 1.2) and 18 were downregulated (WT/p94KI > 1.2) in p94KI mice compared with WT (Supplemental Figure 10). DNA microarray analysis showed that mRNA levels of these proteins were unchanged with a few exceptions (Supplemental Figure 10 and Supplemental Table 1), indicating that these changes were largely at the protein level. Surprisingly, the amounts of all of proteins upregulated in p94KI were increased (or unchanged), and not decreased, in WT-ex mice compared with WT with only one exception; in contrast, proteins downregulated in $\mathrm{p} 94 \mathrm{KI}$ were decreased (or unchanged) in WT-ex (compare p94KI/WT and WT-ex/WT in Supplemental Figure 10). This synchronicity strongly suggests that molecular conditions in the cytosol of cells that have been altered by p94:C129S mutation are nearly identical to those in the WT-ex mice. To reveal the underlying molecular mechanisms, we classified the expression profiles as indicated in Table 1 by focusing on proteins that responded to exercise differentially in WT and p94KI mice, i.e., categories [WT $\uparrow$ and p94KI $\rightarrow$ or $\downarrow$ ] and [WT $\downarrow$ and p94KI $\uparrow$ or $\rightarrow$ ] in Table 1 .

These categories correspond to proteins that are up- or downregulated by exercise in WT mice but that failed to be altered in p94KI mice. Notably, MARP2 and -10 of the 13 identified hsprelated molecules (including 6 small hsp [sHSP], important for muscle cell homeostasis; ref. 19) were included in category [WT $\uparrow$ and $\mathrm{p} 94 \mathrm{KI} \rightarrow$ or $\downarrow$ ]. MARP2 data is consistent with the above Western blot analysis. Other proteins in category [WT $\uparrow$ and p94KI $\rightarrow$ or $\downarrow$ included hexokinase 2 , a rate-limiting enzyme for glycolysis, factors involved in protein translation, and proteasome subunits, which may contribute to the turnover of muscle cells (see Supplemental Figure 10 legend). These results suggest that p94 protease activity regulates the levels of the proteins in categories [WT $\uparrow$ and p94KI $\rightarrow$ or $\downarrow$ ] and [WT $\downarrow$ and $\mathrm{p} 94 \mathrm{KI} \uparrow$ or $\rightarrow$ ] with exercise.

Altogether, our results support the idea that p94 plays an important role in MD pathogenesis, particularly in the exercise-mediated exacerbation of MD, through its dynamic spatial configuration-dependent regulation of MARP2 expression and protein-level upregulation of hsp.

\section{Discussion}

Using p94KI mice, here we present the first direct and conclusive evidence that the loss of $\mathrm{p} 94$ protease activity causes LGMD2A, which is aggravated by age or exercise. We showed that in myocytes, the loss of p94 protease activity causes (a) a slow exchange rate of p94 molecules at the M-line, (b) sluggish accumulation of p94 at the N2A region in response to sarcomeric extension, and (c) impaired upregulation of MARP2 and hsp levels in response to physical stress. Our results support the idea that $\mathrm{p} 94$ protease activity plays a key role in the pathogenesis of LGMD2A. 
A
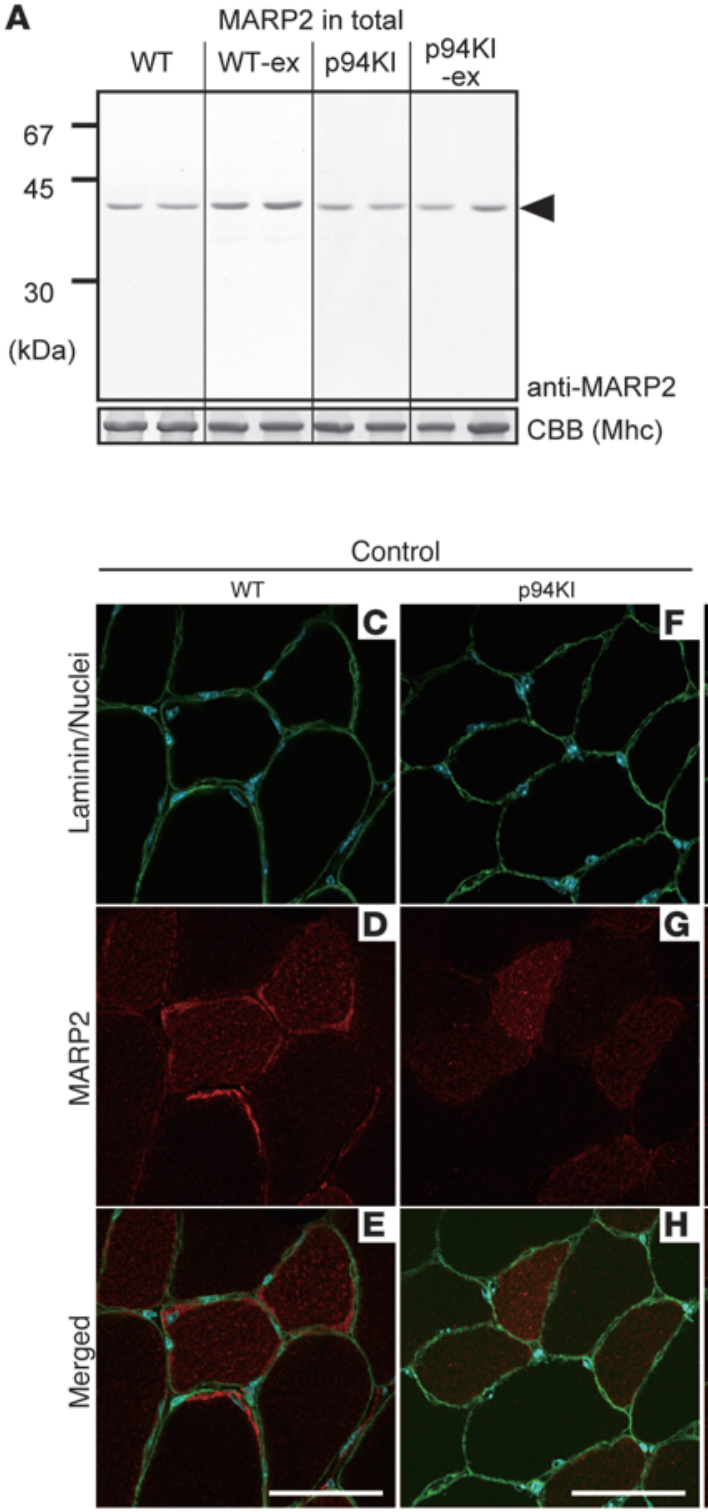

B

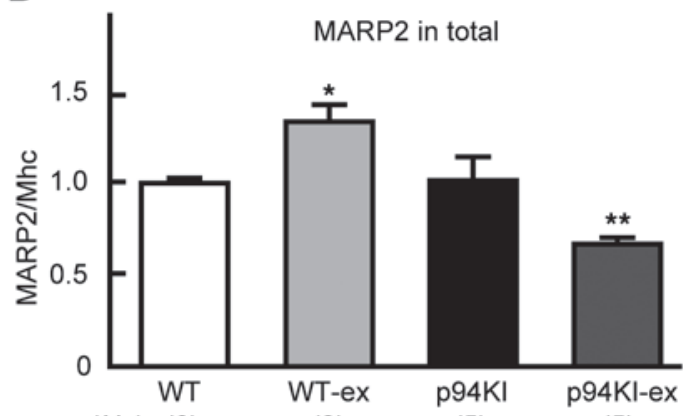

$(N=)(6)$

(5)

(5)

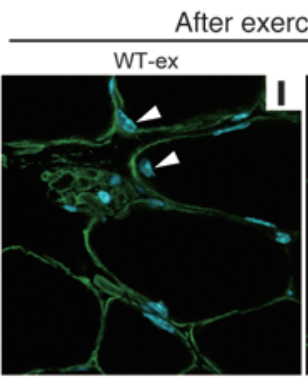

After exercise (day 0)
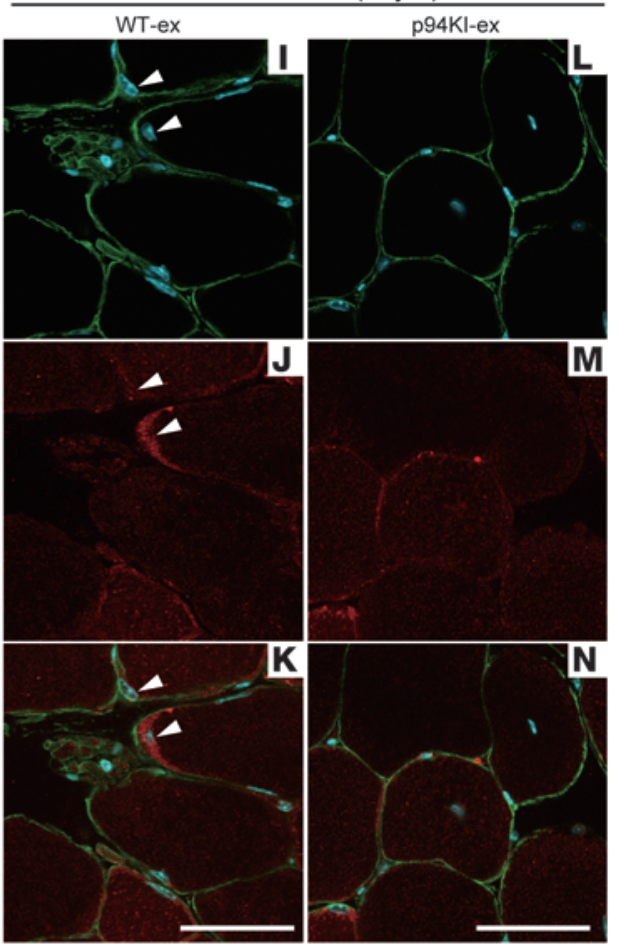

\section{Figure 4}

Changes in MARP2 in WT and p94KI mice before and after exercise. (A and B) MARP2 protein detected in WT, WT-ex, p94KI, and p94KI-ex mice. The total extract of TA muscles was subjected to Western blot analysis using an anti-MARP2 Ab (representative blots are shown; see Supplemental Figure 7 for all the blots). In WT-ex and p94KI-ex mice, the muscles were isolated immediately after exercise. Hairlines indicate lanes that were run on the same gel but were noncontiguous. The full-length MARP2 bands (40 kDa, black arrowheads) were quantified (B) by normalizing them to the CBB-stained band for myosin heavy chain (Mhc) (CBB in A). ${ }^{*} P<0.05$ versus WT; ${ }^{*} P<0.05$ versus p94KI. (C-N) Induction of MARP2 in WT mice after exercise. Muscle cross-sections from WT (C-E and I-K), and p94KI (F-H and L-N) mice were stained with anti-laminin $\alpha 2(\mathbf{C}, \mathbf{F}, \mathbf{I}$, and L) and anti-MARP2 (D, G, J, and M) Abs. The WT-ex and p94KI-ex muscle samples were isolated immediately after exercise (I-N). Nuclei were visualized with DAPI (light blue in C, E, F, H, I, K, L, and N). Note that MARP2-positive myonuclei were detected in the exercised WT (arrowheads) sample. For observations of longitudinal sections and the specificity of the anti-MARP2 Ab, see Supplemental Figure 8 . Scale bars: $50 \mu \mathrm{m}$.

Consistent with our previous in vitro observations (19), p94 changed its in vivo location from the M-line to the N2A region as sarcomeres lengthened, indicating that it undergoes a sarcomere-length-dependent redistribution in vivo. Importantly, the swiftness of p94's redistribution was dependent on its protease activity. Furthermore, our FRAP study showed that the M-line/ cytosol exchange rate (recovery $t_{1 / 2}$ ) of the p94:C129S molecules was approximately twice as slow as that of WT p94. These find- ings indicate that p94 responds to the physical stress of myocytes and that the mobility of p94 is regulated/facilitated by its protease activity.

How is p94's protease activity involved in the regulation of mobility? It should be noted that p94's protease activity is involved in both substrate degradation and autolysis. The activation of p94 causes a limited processing of p94 itself (autoprocessing), resulting in a conformationally altered molecule that is still proteolytically 

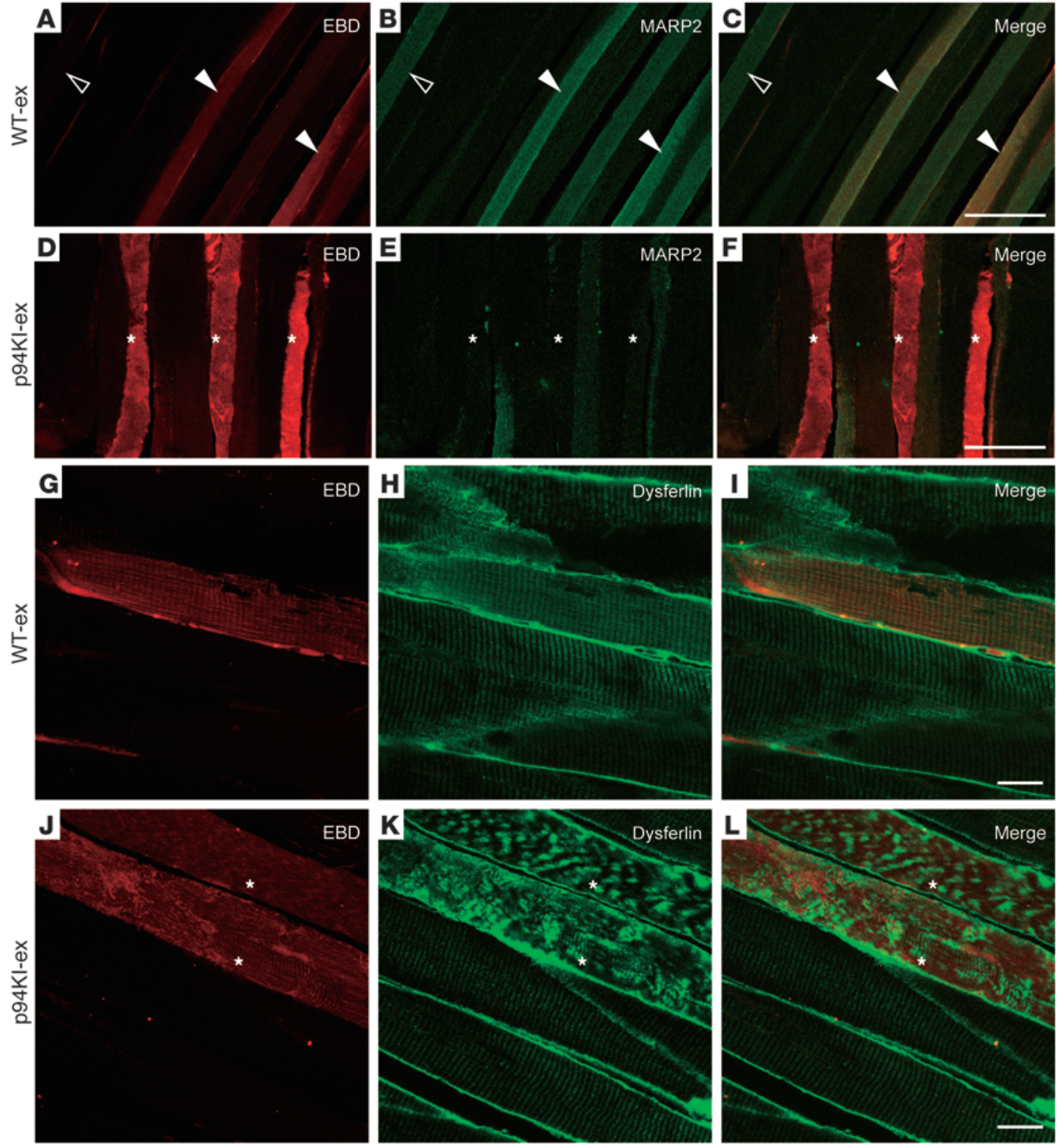

\section{Figure 5}

EBD uptake in the myofibers of exercised WT and p94KI mice. EBD-injected WT and p94KI mice were subjected to downhill running and then sacrificed immediately after exercise. Longitudinal cryostat sections of extensor digitorum longus (EDL) muscles were visualized with EBD autofluorescence (A, D, G, and J), an anti-MARP2 Ab (B and E), or an anti-dysferlin Ab ( $\mathbf{H}$ and $\mathbf{K})$. Merged images are shown at right (C, $\mathbf{F}, \mathbf{I}$, and $\mathbf{L}$ ). The uptake of EBD by myofibers was observed in both the WT-ex and p94KI-ex samples. (A-C) In the WT-ex mice, the EBD-positive myofibers had high MARP2 signals (white arrowheads), but not all the myofibers with high MARP2 signals were EBD positive (open arrowheads). (D-F) In the p94KI-ex mice, the myofibers with EBD uptake showed lower MARP2 signals (asterisks) than those from WT mice and were degenerating (see below). (G-I) In WT-ex mice, normal dysferlin staining (sarcolemma and striation) was observed in the EBD-positive myofibers, indicating that they were not degenerating $(29,30)$. (J-L) In the p94KI-ex mice, the EBD-positive myofibers showed abnormal dysferlin localization, indicating that the sarcolemma was disrupted and the fibers were degenerating (asterisks). Scale bars: $100 \mu \mathrm{m}(\mathbf{A}-\mathbf{F}) ; 20 \mu \mathrm{m}(\mathbf{G}-\mathbf{L})$.

competent $(25,31-33)$. In fact, the proteolytic activity-dependent autoprocessing of p94 is detectable in exercised muscles as a faster migrating fragment of ca. $60 \mathrm{kDa}$ (see Figure 2A). Therefore, the protease activity-dependent conformational changes in p 94 might be important for $\mathrm{p} 94$ 's proper dynamics within the sarcomere.

MARP2 is thought to be involved in stress-response pathways in skeletal muscles $(17,26,28,34,35)$. The importance of MARP2's induction for membrane repair is also supported by recent studies showing that MARP2-overexpressing muscle cells express elevated levels of genes for muscle repair systems, among others (36). Thus, the failure of MARP2 induction in p94KI-ex mice probably compromises the muscle's adaptation to physical stress. We previously showed that p94 competes with MARP2 for binding to the N2A region and that WT p94 is better able to abrogate the MARP2-N2A interaction than p94:C129S (17). Therefore, it is conceivable that the stretch-induced dynamic 
Table 1

Protein expression profiles of WT and p94KI skeletal muscle before and after eccentric exercise

\begin{tabular}{|c|c|c|c|c|c|c|c|c|c|}
\hline GI no. & Protein name & Gene & $\% \operatorname{Cov}$ & WT-ex /WT & $P$ value & p94KI-ex /p94KI & $P$ value & p94KI /WT & $P$ value \\
\hline \multicolumn{10}{|c|}{$[W T \uparrow \text { and } p 94 \mathrm{KI} \uparrow]^{\mathrm{A}}$} \\
\hline 19387852 & DNA mismatch repair protein; MutL homolog & Mlh1 & 1.3 & 1.21 & $1.6 \times 10^{-01}$ & 1.95 & $1.1 \times 10^{-02}$ & 1.00 & $9.4 \times 10^{-01}$ \\
\hline 29294760 & Ribosomal protein S6 kinase $\alpha-1$ & Rps6ka1 & 2.6 & 1.81 & $4.5 \times 10^{-02}$ & 1.70 & $4.0 \times 10^{-01}$ & 2.06 & $9.0 \times 10^{-02}$ \\
\hline 37359988 & Brain-specific angiogenesis inhibitor 3 & Bai3 & 2.0 & 1.64 & $9.3 \times 10^{-02}$ & 1.48 & $1.7 \times 10^{-01}$ & 1.84 & $2.2 \times 10^{-03}$ \\
\hline 313103 & Myomesin, skelemin & Myom1 & 1.6 & 1.59 & - & 1.31 & - & 1.44 & - \\
\hline 74220201 & Ubiquitin conjugating enzyme E2L3, UbcH7 & Ube2/3 & 10.7 & 1.70 & - & 1.24 & - & 1.71 & - \\
\hline 74186554 & Nedd8 & Nedd8 & 25.0 & 1.39 & - & 1.20 & - & 1.42 & - \\
\hline 74178118 & Transferrin & Trf & 18.9 & 1.35 & $5.0 \times 10^{-04}$ & 1.20 & $1.8 \times 10^{-02}$ & 1.01 & $8.9 \times 10^{-01}$ \\
\hline 28302235 & 5'-to-3' DNA helicase homolog & Pif1 & 2.3 & 1.40 & - & 1.16 & - & 1.42 & - \\
\hline 29244166 & SH3 and cysteine-rich domain 3 & Stac3 & 6.4 & 1.55 & - & 1.14 & - & 1.48 & - \\
\hline 58477305 & Muscle-restricted coiled-coil protein & Murc & 4.0 & 1.44 & $3.0 \times 10^{-02}$ & 1.14 & $6.1 \times 10^{-02}$ & 1.11 & $5.5 \times 10^{-01}$ \\
\hline 6753912 & Ferritin heavy chain 1 & Fth1 & 8.2 & 1.61 & - & 1.13 & - & 1.29 & - \\
\hline 149257950 & 28S Ribosomal protein S36, S36mt & Mrps36 & 17.6 & 1.33 & - & 1.12 & - & 1.86 & - \\
\hline 74223108 & Dihydrolipoamide dehydrogenase & Dld & 13.6 & 1.36 & $3.1 \times 10^{-02}$ & 1.10 & $1.4 \times 10^{-01}$ & 1.29 & $1.8 \times 10^{-02}$ \\
\hline \multicolumn{10}{|c|}{$[\mathrm{WT} \uparrow \text { and p94KI } \rightarrow \text { or } \downarrow]^{\mathrm{B}}$} \\
\hline 21304450 & Immunoglobulin gamma 1 heavy chain & lghg1 & 5.0 & 2.66 & $6.8 \times 10^{-02}$ & 0.97 & $8.6 \times 10^{-01}$ & 1.31 & $2.2 \times 10^{-01}$ \\
\hline 40644653 & Kinesin family member 15 & Kif15 & 1.3 & 2.53 & $4.5 \times 10^{-03}$ & 1.07 & $3.1 \times 10^{-01}$ & 2.87 & $1.2 \times 10^{-02}$ \\
\hline 74151744 & Proteasome subunit, $\alpha 4$ & Psma4 & 4.2 & 1.72 & $1.8 \times 10^{-01}$ & 1.05 & $7.9 \times 10^{-01}$ & 1.45 & $2.9 \times 10^{-01}$ \\
\hline 76779273 & Chaperonin, HSP60, HSPD1 & Hspd1 & 2.7 & 1.65 & - & 1.06 & - & 1.64 & - \\
\hline 51890211 & Leu-rich repeat containing 8 family $E$ & Lrrc8e & 1.3 & 1.60 & $6.4 \times 10^{-02}$ & 0.81 & $6.2 \times 10^{-01}$ & 1.55 & $2.3 \times 10^{-01}$ \\
\hline 13507646 & Heat shock protein 8, SHSPB8 & Hspb8 & 12.2 & 1.59 & - & 0.79 & - & 3.60 & - \\
\hline 31542970 & Cardiovascular heat shock protein & Hspb7 & 7.7 & 1.59 & $1.9 \times 10^{-02}$ & 0.76 & $3.8 \times 10^{-02}$ & 1.30 & $8.0 \times 10^{-02}$ \\
\hline 6754724 & Proteasome $26 \mathrm{~S}$ subunit, non-ATPase, 7 & Psmd7 & 6.2 & 1.55 & $2.1 \times 10^{-01}$ & 0.85 & $7.6 \times 10^{-01}$ & 1.24 & $7.9 \times 10^{-01}$ \\
\hline 7305143 & Hexokinase 2 & Hk2 & 0.9 & 1.47 & $7.2 \times 10^{-02}$ & 1.09 & $5.2 \times 10^{-01}$ & 1.70 & $1.7 \times 10^{-01}$ \\
\hline 74147026 & Heat shock protein $90 \mathrm{kDa} \alpha$, class B1 & Hsp90ab1 & 15.2 & 1.44 & $1.7 \times 10^{-03}$ & 0.92 & $2.1 \times 10^{-01}$ & 1.42 & $7.3 \times 10^{-03}$ \\
\hline 548879 & Ras suppressor protein 1 (Rsu-1) (RSP-1) & Rsu1 & 6.1 & 1.42 & - & 0.67 & - & 1.71 & - \\
\hline 82795783 & Eukaryotic translation elongation factor $1 \gamma, \mathrm{eEF}-1 \gamma$ & Eef1g & 2.6 & 1.40 & $5.3 \times 10^{-01}$ & 0.87 & $8.8 \times 10^{-02}$ & 1.39 & $6.3 \times 10^{-01}$ \\
\hline 148685426 & Tu translation elongation factor, mitochondrial & Tufm & 8.3 & 1.38 & $5.1 \times 10^{-02}$ & 1.07 & $7.1 \times 10^{-01}$ & 1.39 & $8.1 \times 10^{-02}$ \\
\hline 6753530 & Crystallin, $\alpha \mathrm{B}$ & Cryab & 48.6 & 1.33 & $1.1 \times 10^{-03}$ & 0.76 & $6.0 \times 10^{-04}$ & 1.29 & $6.8 \times 10^{-03}$ \\
\hline 158937312 & Heat shock protein 1, SHSPB1 & Hspb1 & 48.8 & 1.30 & $1.2 \times 10^{-02}$ & 0.72 & $3.8 \times 10^{-03}$ & 1.37 & $2.6 \times 10^{-02}$ \\
\hline 74224118 & Radixin isoform a & $R d x$ & 6.8 & 1.28 & $1.7 \times 10^{-01}$ & 1.07 & $4.6 \times 10^{-01}$ & 1.28 & $2.1 \times 10^{-01}$ \\
\hline 74177941 & Hsc70 interacting protein & St13 & 3.2 & 1.28 & $4.9 \times 10^{-02}$ & 0.86 & $1.4 \times 10^{-01}$ & 1.36 & $6.9 \times 10^{-02}$ \\
\hline 59958370 & Heat shock protein, $\alpha$-crystallin-related, B6 & Hspb6 & 14.2 & 1.23 & $1.5 \times 10^{-02}$ & 0.91 & $8.1 \times 10^{-02}$ & 1.27 & $4.2 \times 10^{-02}$ \\
\hline 26380702 & Chaperone, ABC1 activity of bc1 complex like & Cabc1 & 1.4 & 1.23 & $1.3 \times 10^{-01}$ & 0.85 & $1.6 \times 10^{-02}$ & 1.16 & $3.6 \times 10^{-01}$ \\
\hline 74147276 & Proteasome $26 \mathrm{~S}$ subunit, ATPase 2 & Psmc2 & 3.0 & 1.21 & $5.8 \times 10^{-01}$ & 0.98 & $8.1 \times 10^{-01}$ & 0.95 & $8.2 \times 10^{-01}$ \\
\hline 61098127 & Heat shock protein 2, sHSPB2 & Hspb2 & 10.4 & 1.19 & $8.1 \times 10^{-01}$ & 1.08 & $3.7 \times 10^{-02}$ & 1.05 & $6.3 \times 10^{-01}$ \\
\hline 122889668 & Alanyl-tRNA synthetase domain containing 1 & Aarsd1 & 1.8 & 1.10 & $2.0 \times 10^{-02}$ & 1.08 & $6.5 \times 10^{-01}$ & 1.10 & $5.1 \times 10^{-01}$ \\
\hline \multicolumn{10}{|c|}{$[\mathrm{p} 94 \mathrm{KI} \uparrow \text { or } \downarrow \text { and } \mathrm{WT} \rightarrow]^{\mathrm{A}}$} \\
\hline 54607128 & Programmed cell death protein 11, ALG-4 & Pdcd11 & 0.8 & 1.00 & $9.7 \times 10^{-01}$ & 1.45 & $3.4 \times 10^{-02}$ & 0.86 & $7.0 \times 10^{-02}$ \\
\hline 31982861 & Carbonic anhydrase 3 & Car3 & 59.2 & 1.01 & $7.4 \times 10^{-01}$ & 1.26 & $1.7 \times 10^{-16}$ & 0.90 & $1.1 \times 10^{-02}$ \\
\hline 2340058 & Troponin T & Tnnt3 & 29.2 & 1.09 & $4.7 \times 10^{-02}$ & 0.90 & $3.0 \times 10^{-03}$ & 1.20 & $2.8 \times 10^{-03}$ \\
\hline 156257677 & $\beta$-Globin & $H b b-b 1$ & 44.9 & 0.97 & $2.9 \times 10^{-01}$ & 0.89 & $1.2 \times 10^{-07}$ & 1.12 & $1.9 \times 10^{-02}$ \\
\hline 74204028 & Dihydrolipoamide S-succinyltransferase & Dlst & 7.9 & 1.00 & $9.9 \times 10^{-01}$ & 0.87 & $1.7 \times 10^{-03}$ & 1.01 & $9.9 \times 10^{-01}$ \\
\hline 82617575 & Glutamyl-prolyl-tRNA synthetase & Eprs & 1.8 & 0.98 & $8.4 \times 10^{-0} 01$ & 0.86 & $3.0 \times 10^{-02}$ & 0.94 & $5.0 \times 10^{-01}$ \\
\hline 6678391 & Troponin I, skeletal, fast 2 & Tnni2 & 55.5 & 0.98 & $7.8 \times 10^{-01}$ & 0.83 & $3.0 \times 10^{-04}$ & 1.22 & $1.4 \times 10^{-01}$ \\
\hline 74147564 & Nicotinamide phosphoribosyltransferase & Nampt & 1.6 & 1.05 & $7.8 \times 10^{-01}$ & 0.82 & $3.5 \times 10^{-02}$ & 1.21 & $5.1 \times 10^{-01}$ \\
\hline \multicolumn{10}{|c|}{$[W T \downarrow \text { and } p 94 K I \uparrow \text { or } \rightarrow]^{B}$} \\
\hline 50510361 & Peroxiredoxin 6 & $\operatorname{Prdx6}$ & 8.4 & 0.59 & - & 1.22 & - & 0.40 & - \\
\hline 6753290 & Calsequestrin 1 & Casq1 & 14.0 & 0.90 & $1.4 \times 10^{-02}$ & 1.28 & $1.2 \times 10^{-07}$ & 0.83 & $3.4 \times 10^{-04}$ \\
\hline 6671762 & Creatine kinase, muscle & $C k m$ & 90.0 & 0.88 & $1.4 \times 10^{-15}$ & 1.07 & $8.7 \times 10^{-18}$ & 0.92 & $1.5 \times 10^{-04}$ \\
\hline 74144566 & DnaJ (Hsp40) homolog, subfamily B14 & Dnajb14 & 5.1 & 0.77 & $9.4 \times 10^{-08}$ & 1.01 & $6.2 \times 10^{-02}$ & 0.74 & $2.8 \times 10^{-07}$ \\
\hline 31982522 & Acyl-coenzyme A dehydrogenase, short chain & Acads & 8.3 & 0.83 & $1.7 \times 10^{-01}$ & 1.01 & $3.9 \times 10^{-01}$ & 0.80 & $1.6 \times 10^{-02}$ \\
\hline 84794552 & Phosphatidylethanolamine-binding protein 1 & Pebp1 & 46.5 & 0.82 & $1.2 \times 10^{-02}$ & 1.00 & $9.6 \times 10^{-01}$ & 0.70 & $6.7 \times 10^{-03}$ \\
\hline 31980767 & Parvalbumin & Pvalb & 88.2 & 0.79 & $3.5 \times 10^{-07}$ & 0.99 & $7.0 \times 10^{-01}$ & 0.69 & $3.6 \times 10^{-12}$ \\
\hline 7949078 & Myosin regulatory light chain 2 , fast skeletal muscle & Mylpf & 13.6 & 0.82 & $7.3 \times 10^{-02}$ & 0.97 & $7.0 \times 10^{-01}$ & 0.82 & $2.5 \times 10^{-02}$ \\
\hline 74225421 & ATP synthase subunit $\beta$, mitochondrial & Atp5b & 19.7 & 0.86 & $2.0 \times 10^{-02}$ & 0.97 & $5.7 \times 10^{-01}$ & 0.91 & $2.7 \times 10^{-01}$ \\
\hline 148709839 & Insulin degrading enzyme & Ide & 0.7 & 0.82 & $1.4 \times 10^{-02}$ & 0.93 & $1.1 \times 10^{-01}$ & 0.92 & $1.7 \times 10^{-01}$ \\
\hline 13385680 & 2,4-Dienoyl CoA reductase 1 , mitochondrial & Decr1 & 4.5 & 0.66 & $6.8 \times 10^{-02}$ & 0.92 & $1.1 \times 10^{-02}$ & 0.59 & $9.6 \times 10^{-02}$ \\
\hline 21359820 & Myoglobin & $M b$ & 85.7 & 0.78 & $9.3 \times 10^{-19}$ & 0.92 & $4.7 \times 10^{-03}$ & 0.64 & $2.6 \times 10^{-27}$ \\
\hline \multicolumn{10}{|c|}{$[W T \downarrow \text { and } p 94 \mathrm{KI} \downarrow]^{\mathrm{A}}$} \\
\hline 29789016 & Fast skeletal myosin alkali light chain $1 f$ & Myl1 & 49.5 & 0.80 & $9.0 \times 10^{-05}$ & 0.82 & $4.0 \times 10^{-05}$ & 0.89 & $7.8 \times 10^{-03}$ \\
\hline 171906557 & Peptidyl arginine deiminase, type II & Padi2 & 5.6 & 0.88 & $4.7 \times 10^{-02}$ & 0.80 & $6.2 \times 10^{-03}$ & 0.93 & $2.2 \times 10^{-01}$ \\
\hline 6678371 & Fast skeletal muscle troponin C & Tnnc2 & 7.5 & 0.64 & $7.7 \times 10^{-02}$ & 0.74 & $4.1 \times 10^{-01}$ & 0.84 & $2.1 \times 10^{-01}$ \\
\hline
\end{tabular}

Relative protein amounts of WT, WT-ex, p94KI, and p94KI-ex mice were analyzed by iTRAQ-labeling, 2D-LC-MALDI-TOF/TOF, and ProteinPilot. Proteins that have \%Cov (ratio of a sum of unique aa residues in the detected peptides to the number of aa residues of the full-length protein) of nonzero value and show WT-ex/WT $>1.1$ or $<0.91$, p94KI-ex/ p94KI $>1.1$ or $<0.91$, or $P<0.05$ are listed here with classification of 5 categories (defined after exercise) as indicated. Definitions of categories are as follows: [WT $\uparrow$ and p94KI $\uparrow$, WT-exWT and p94KI-ex/p94KI > 1.1; [WT $\uparrow$ and p94KI $\rightarrow$ or $\downarrow]$, WT-ex/WT > 1.1 and p94KI-ex/p94KI < 1.1; [p94KI $\uparrow$ or $\downarrow$ and WT $\rightarrow$ ], $1.1>$ WT-ex/WT > 0.91 and [p94KI-ex/p94KI $>1.1$ or < 0.91]; [WT $\downarrow$ and p94KI $\uparrow$ or $\rightarrow$ ], WTMT-ex > 1.1 and p94KI/p94KI-ex < 1.1; [WT $\downarrow$ and p94KI $\downarrow$ ] WT/WT-ex and p94KI/p94KI-ex > 1.1. $\uparrow$, upregulated; $\downarrow$, downregulated; $\rightarrow$, unchanged. Bold text indicates hsp; underlined text indicates small hsp. ${ }^{\text {AS }}$ orted by p94KI-ex/p94KI values. ${ }^{B}$ Sorted by WT-ex/WT values. Data shown here are from the same data set shown in Supplemental Figure 10; refer to its legend for details. 

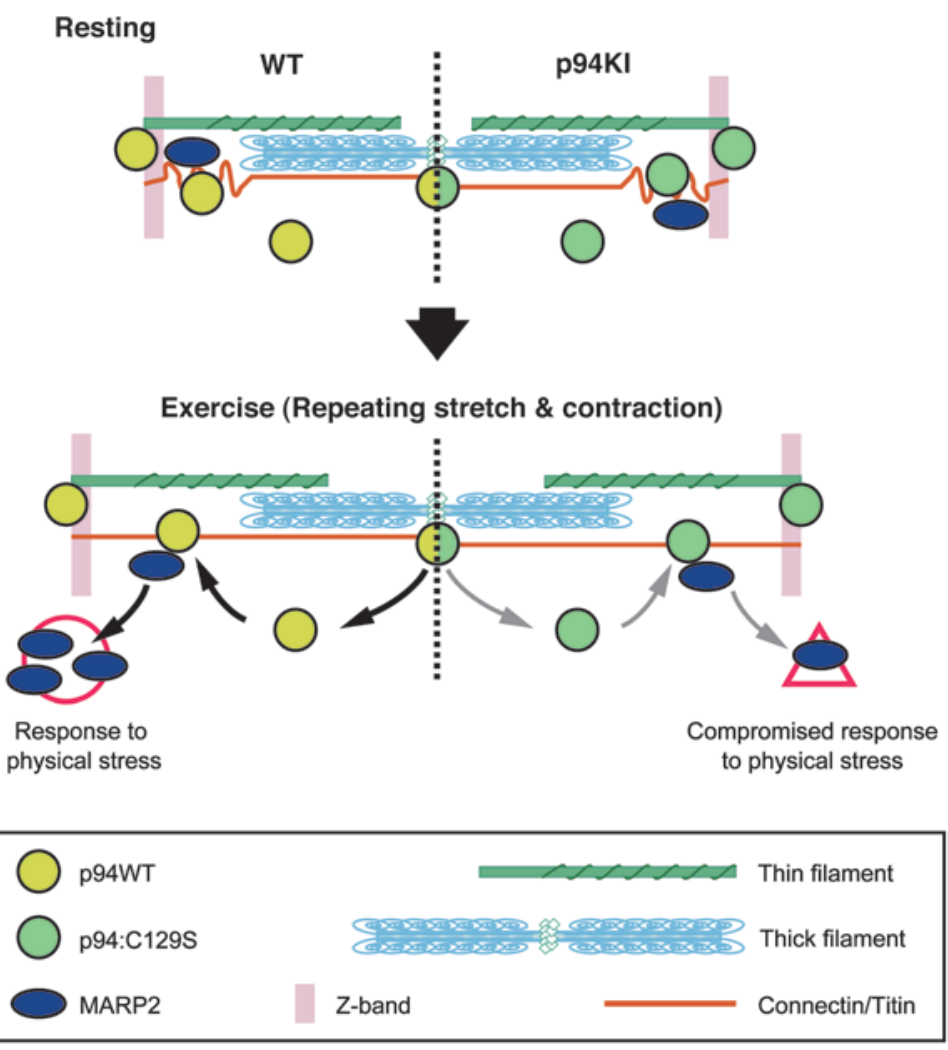

\section{Figure 6}

A model for the p94-mediated signal response to physical stress. In WT mice, when skeletal muscles undergo physical stress such as exercise, p94WT accumulates at the N2A region and MARP2 translocates from the N2A region to the myonuclei to promote the adaptive response to stress. In contrast, in the p94KI mice, in response to physical stress, the p94:C129S protein accumulates slowly at the $\mathrm{N} 2 \mathrm{~A}$ region, and the MARP2 induction is lower than in WT mice. Consequently, MARP2 signaling does not function properly in the $\mathrm{p} 94 \mathrm{KI}$ mice. Thus, the $\mathrm{p} 94$ protease activity is required for the adaptive response to physical stress in skeletal muscles. Black and gray arrows indicate the proper and compromised mobility in response to physical stress in WT and p94KI mice, respectively. redistribution of $\mathrm{p} 94$ from the $\mathrm{M}$-line to the $\mathrm{N} 2 \mathrm{~A}$ region modulates the dissociation of MARP2 from connectin/titin and the subsequent translocation of MARP2 to the nucleus to transmit signals of mechanical perturbation and that this process is compromised in p94KI mice (Figure 6). In other words, the loss of p94 protease activity may disrupt a MARP2-mediated stressresponse pathway, resulting in muscle degeneration. This notion is also supported by the observations that exercise induces p94 proteolytic activity and the upregulated transcription of MARP2 in human skeletal muscles $(20,27)$ and that p94 is mislocalized in LGMD2A patients (37).

We noted a striking similarity in the proteomic profiles of muscle cytosol in p94KI and WT-ex mice, including upregulation of hsp, likely indicating that the skeletal muscle of p94KI mice is in a permanent stress state like that caused by eccentric exercise in WT mice. Conversely, the very mild symptoms of p94KI mice can be ascribed to the upregulation of hsp. Upon exercise, since p94 translocates to the cytosol, the relative amounts of p94 in the M-line decrease. Indeed, our iTRAQ experiments detected an approximately 1.2- to 1.6-fold increase in myomesin 1 and 2, the major proteins in the M-line, in WT-ex and p94KI mice, suggesting that p94 is one of the proteases responsible for myomesin degradation. In the cytosol, muscle type CK (CK-M) is a candidate target for $\mathrm{p} 94$ proteolysis since CK-M decreases with exercise in WT mice, but does not significantly change in p94KI mice. On the other hand, in WT mice, exercise upregulated proteins involved in protein turnover, e.g., ubiquitin-related molecules (UbcH7, Nedd8), proteasome subunits (PSMA4, C2, D7), and proteins related to translation (S36mt, EF-Tu, eEF-1 $\gamma, \mathrm{RSK}-1)$. This can be explained by the change in p94 localization and/or by hypothesizing that its degrading protease or proteases are downregulated by $\mathrm{p} 94$. Some of these proteins (proteasome subunits, EF-Tu, and eEF-1 $\gamma$ ) are not upregulated or are even downregulated in p94KI-ex mice, suggesting that they are under the control of $\mathrm{p} 94$ protease activity.

Although CK levels are elevated in LGMD2A patients with a CAPN3-null mutation, in $\mathrm{p} 94 \mathrm{KO}$ mice, and in patients with other MDs $(3,11,13)$, our p94KI mice did not exhibit an elevated CK value when unexercised. Similarly, some LGMD2A patients that express an apparently normal-sized p94 protein with missense mutations, such as P359L, show almost normal CK values (38). Considering that $\mathrm{P} 359 \mathrm{~L}$, which is adjacent to one of the active-site residues (N358), is likely to impair the p94 protease activity, these findings suggest that a novel function of $\mathrm{p} 94$, not its protease activity, is involved in the maintenance of the sarcolemma. Further studies will be required to elucidate this function, which may be facilitated by comparing the p94KI mice with the p94KO mice.

Previous studies have demonstrated that the protein dynamics of the highly organized sarcomere are important for the sarcomere's structural integrity (39). Given that p94KI-ex showed an exacerbated MD phenotype, we suggest that the decreased ability of inactive p94 to swiftly change its distribution in response to physical stress results in a delayed or inadequate adaptive response of the myocytes to physical stress, which contributes to MD pathogenesis. Thus, it is plausible that p94 functions as a sensor that detects physical stress through stretch-dependent autoprocessing. Since skeletal muscles are exceptionally adaptable to a variety of stimuli (exercise, denervation, starvation, hypoxia, invasive surgery, trauma, etc.), they must require cellular machinery for the qualitative and quantitative sensing of these stimuli. In this context, p94 is an excellent candidate as a sensor for the intensity of physical stress. Our results demonstrating the importance of spa- 
tiotemporally regulated p94 behavior in the protective response of skeletal muscles provide what we believe are novel and important insights into the complex pathogenesis of LGMD2A as well as ideas for the development of future therapeutic strategies. In addition, our observations may be important for understanding how physical stress in skeletal muscles is connected to adaptive cellular responses, although the precise molecular mechanisms should be further investigated.

\section{Methods}

Experimental animals. All procedures using experimental animals were approved by the Experimental Animal Care and Use Committee of Rinshoken, and the animals were treated according to the committee's guidelines. C57BL/6 mice were purchased from Nihon CLEA Inc. Cre-recombinaseexpressing $\mathrm{Tg}$-mice were provided by the Jackson Laboratory.

Construction of the targeting vector and generation of $p 94 K I$. The missense C129S mutation and a neomycin-resistance gene (neoR) flanked by loxP sequences were introduced into exon 3 and intron 3 , respectively, of the Capn 3 gene in mouse ES cells by electrotransfection of the targeting vector (Supplemental Figure 1A). The recombinant ES cells were used for blastocyst injection to produce germline-transmitting chimeric mice. F1 heterozygotes with the proper recombination were crossed with $\mathrm{Tg}$ mice expressing Cre recombinase in their germline cells. The resultant mice were confirmed by Southern blot analysis to have the C129S mutation without $n e o R$ and were backcrossed with C57BL/6J mice for more than 8 generations (see Supplemental Figure 1 for details).

Mouse running protocol and serum $C K$ measurement. An exercise protocol was designed as previously reported (40) with slight modification. Briefly, WT and p94KI mice (15 to 18 weeks old) were made to run on motor-driven treadmills (MK-680S; Muromachi Inc.). The running program was as follows: $\left(16^{\circ}\right.$ decline, $\left.18 \mathrm{~m} / \mathrm{min} \times 20 \mathrm{~min}\right) \times 3$ sets with 3 -minute intervals between each set. Muscle and blood samples were taken immediately after the exercise unless otherwise stated. For the EBD assay, 2\% EBD in PBS was injected intravenously immediately after exercise ( $10 \mu \mathrm{l} / \mathrm{g}$ body weight) (41), and the mice were sacrificed the next day. Since this protocol showed almost no EBD-stained myofibers in WT mice, in another set of experiments, EBD was preinjected before exercise, and the mice were sacrificed immediately afterwards. The dissected muscles were immediately frozen in liquid nitrogen-cooled isopentane.

For the serum CK measurement, blood was taken from the heart under anesthesia. To prevent nonspecific increases in the CK value, a single blood draw per sample was used for quantification. Serum CK was measured by SRL Inc.

Histology and Western blot analysis. H\&E staining and immunochemical labeling were performed as previously reported (42). Fluorescence was observed on a laser-scanning confocal microscope (Zeiss LSM510). The images were manipulated with LSM imaging software. The number of myofibers and CNM were counted in H\&E-stained sections. The CSArs of myofibers were measured with AlphaEaseFC Software ver. 3.1.2 (Alpha InnoTech Corp.). Western blot analysis was performed as previously described (19). Skeletal muscles were lysed with TED buffer (10 mM Tris/ $\mathrm{Cl}$ [pH 7.5], 1 mM EDTA, 1 mM DTT). The Sup and Ppt fractions were prepared by centrifugation at $23,000 \mathrm{~g}$ for 20 minutes. The intensity of the bands was normalized to that of the CBB-stained actin or Mhc band. The primary Abs used in this study are listed in Supplemental Table 2.

Quantification of the $p 94$ signal in sarcomeres. After staining with the antipNS Ab, which detects p94 (43), the average signal intensities of p94 in the $\mathrm{M}$ and N2A zones were quantified with Photoshop CS2 (Adobe). The p94 signal ratio in a single sarcomere was calculated as the ratio of the p94 signal density in $\mathrm{M}$ to the p94 signal density in N2A. Each value was plotted on a scatter graph in relation to the sarcomeric length, which was measured as the distance between the centers of adjacent s- $\alpha$-actinin-positive Z-lines with LSM imaging software. The total number of sarcomeres counted was 195 for WT and 188 for p94KI.

EMstudy. EDL muscles were fixed with $2 \%$ glutaraldehyde, postfixed with $1 \% \mathrm{OsO}_{4}$, and embedded in Epon. Ultrathin sections were stained with uranyl acetate and examined using a JEM 1200EX transmission electron microscope (JEOL).

Muscle culture, transfection, and FRAP. The preparation of mouse skeletal muscle primary culture cells and transfection were carried out as previously described (19). The cDNAs for full-length human p94 and its protease-inactive form p94:C129S were cloned into the pEGFP vector (Clontech). Five days after switching to differentiation medium, myocytes transfected with an expression vector encoding a WT p94-EGFP fusion protein, p94:C129SEGFP fusion protein, or EGFP (pEGFP mock vector) were subjected to FRAP analysis with an LSM510 confocal microscope. The regions of interest were bleached for 30 seconds using LSM software. For control experiments using pEGFP, 30 seconds of photobleaching reduced the EGFP signal by only about $50 \%$, because of the rapid mobility of the EGFP molecules (see Supplemental Figure 5). The FRAP process was recorded using LSM software. For the FRAP experiments using p94-fused EGFP, the relative EGFP intensity was calculated as the ratio of EGFP intensity at each time point to the intensity before bleaching and the $t_{1 / 2}$ for recovery was calculated as reported previously (22). Ten fibers each were quantified for WT $\mathrm{p} 94$ and p94:C129S.

To calculate the mobile fraction $\left(M_{1}, M_{2}, \ldots\right)$ and recovery $t_{1 / 2}$, first, the value of the initial photobleached image $(t=0)$ was subtracted from each subsequent image, and the results were normalized by setting the prebleach intensity to 1 and the intensity of the initial photobleached image $(t=0)$ to 0 . A curve-fitting method was used to extract $M_{1}$ and $t_{1 / 2}$ from the FRAP curve, as previously reported (22). MS-Excel was used to fit the data to the following exponential equation: $r=M_{1}\left(1-\exp \left[-k_{1} t\right]\right)$, where $R$ is the relative recovery of the fluorescence intensity of the bleached area at time $t$, and $M_{1}$ is the mobile fraction of the exponential process with rate constant $k_{1} \cdot t_{1 / 2}$ was calculated as $t_{1 / 2}=0.69315 / k_{1}$. Note that this equation fit the data well, and a dual exponential fit did not improve the fit.

iTRAQ labeling and data analysis. Buffer $(0.5 \mathrm{M}$ triethylammonium bicarbonate, $0.1 \% \mathrm{SDS}$ ) soluble fractions of TA muscles were prepared from unexercised WT and p94KI mice and WT-ex and p94KI-ex mice immediately after exercise. Each sample ( $45 \mu \mathrm{g}$ of protein) was a mixture of equal amounts of protein from 3 individual mice and labeled with iTRAQ-114 (WT), -115 (p94KI), -116 (WT-ex), or -117 (p94KI-ex) reagents according to the manufacturer's instructions (ABI). The samples were subjected to fractionation (1,440 spots) using a DiNa LC system with HiQ-Sil-SCX, HiQSil-C18-3, and a MALDI-plate spotter (KYA Tech) as described previously (44). Spotted fractions were analyzed by MALDI-TOF mass spectrometry (ABI 4800), and proteins were identified using ProteinPilot 2.0 (ABI) and Swiss-Prot Database. ProteinPilot 2.0 software detected 4,965 distinct peptides for 660 proteins from 14,660 MS/MS spectra (confidence > 0.7). See Supplemental Figure 10 for details.

DNA microarray analysis. Poly(A)+ RNA was isolated from TA muscles of WT and p94KI (2 for each). DNA microarray analysis was performed using an Affymetrix DNA chip U74A according to the manufacturer's instructions and as previously described (18).

Statistics. Statistical analyses were performed by the 2-tailed Student's $t$ test to determine statistical significance. $P$ (exact significance) $<0.05$ was considered statistically significant. As for statistical significance of $k\left(=0.69315 / t_{1 / 2}\right)$ described in Figure 3, C and P, several independent analyses were done (see Supplemental Figure 6 for details). Error bars denote SEM. Calculations were performed using Origin 8 Pro (OriginLab Corp.), MS Excel (Microsoft Corp.), and/or "R” program package (45). 


\section{Acknowledgments}

We are grateful to all the members of the Calpain Project (Rinshoken), the Laboratory of Biological Function, and the Laboratory of Molecular Structure and Function (The University of Tokyo) for valuable support and discussions. We thank Choji Taya and Hiromichi Yonekawa of Rinshoken for their excellent advice on mouse manipulation and maintenance and Motoya Katsuki of the National Institute for Basic Biology for assistance in the initial stages of this study. We dedicate this paper to the memory of Koichi Suzuki, who passed away on April 20, 2010. We will always be grateful for his generous and humble mentorship of all of us. This work was supported in part by MEXT.KAKENHI (18076007 to H. Sorimachi; 22770139 to Y. Ono), JSPS.KAKENHI (18700392, 20500369 to K. Ojima; 19658057, 20370055 to H. Sorimachi), by a research grant (20B-13) for nervous and mental disorders from the Ministry of Health, Labor and Welfare, Japan (to H. Sorimachi), by a Takeda Science Foundation research grant (to H. Sorimachi), an NIH grant (062881 to H. Granzier), and the Deutsche Forschungsgemeinschaft (to S. Labeit).

Received for publication July 31, 2009, and accepted in revised form May 19, 2010.

Address correspondence to: Hiroyuki Sorimachi, Calpain Project, Rinshoken, 2-1-6 Kamikitazawa, Setagaya-ku, Tokyo 1568506, Japan. Phone: 81.3.5316.3277; Fax: 81.3.5316.3613; E-mail: sorimachi-hr@igakuken.or.jp.

\section{Koichi Suzuki is deceased.}

1. Tidball JG, Spencer MJ. Calpains and muscular dystrophies. Int J Biochem Cell Biol. 2000;32(1):1-5.

2. Richard I, et al. Mutations in the proteolytic enzyme calpain 3 cause limb-girdle muscular dystrophy type 2A. Cell. 1995;81(1):27-40.

3. Groen EJ, et al. Analysis of the UK diagnostic strategy for limb girdle muscular dystrophy $2 \mathrm{~A}$. Brain. 2007;130(pt 12):3237-3249.

4. Nigro V. Molecular bases of autosomal recessive limb-girdle muscular dystrophies. Acta Myol. 2003;22(2):35-42.

5. Duguez S, Bartoli M, Richard I. Calpain 3: a key regulator of the sarcomere? FEBS $J$. 2006;273(15):3427-3436.

6. Leiden Muscular Dystrophy pages. Leiden Open Variation Database. http://www.dmd.nl/capn3_ seqvar.html. Updated March 28, 2010. Accessed May 13, 2010.

7. Suzuki K, Hata S, Kawabata Y, Sorimachi H. Structure, activation, and biology of calpain. Diabetes 2004;53(suppl):S12-S18.

8. Goll DE, Thompson VF, Li H, Wei W, Cong J. The calpain system. Physiol Rev. 2003;83(3):731-801.

9. Richard I, et al. Calpainopathy-a survey of mutations and polymorphisms. Am J Hum Genet. 1999;64(6):1524-1540.

10. Saenz A, et al. LGMD2A: genotype-phenotype correlations based on a large mutational survey on the calpain 3 gene. Brain. 2005;128(pt 4):732-742.

11. Piluso G, et al. Extensive scanning of the calpain-3 gene broadens the spectrum of LGMD2A phenotypes. J Med Genet. 2005;42(9):686-693.

12. Tagawa K, et al. Myopathy phenotype of transgenic mice expressing active site-mutated inactive p94 skeletal muscle-specific calpain, the gene product responsible for limb girdle muscular dystrophy type 2A. Hum Mol Genet. 2000;9(9):1393-1402.

13. Richard I, et al. Loss of calpain 3 proteolytic activity leads to muscular dystrophy and to apoptosisassociated $\mathrm{IkBa} /$ nuclear factor $\mathrm{kB}$ pathway perturbation in mice. J Cell Biol. 2000;151(7):1583-1590.

14. Kramerova I, Kudryashova E, Tidball JG, Spencer MJ. Null mutation of calpain 3 (p94) in mice causes abnormal sarcomere formation in vivo and in vitro. Hum Mol Genet. 2004;13(13):1373-1388.

15. Sorimachi $\mathrm{H}$, et al. Muscle-specific calpain, p94, responsible for limb girdle muscular dystrophy type $2 \mathrm{~A}$, associates with connectin through IS2, a p94-specific sequence. J Biol Chem. 1995; 270(52):31158-31162.

16. Granzier HL, Labeit S. The giant protein titin: a major player in myocardial mechanics, signaling, and disease. Circ Res. 2004;94(3):284-295.

17. Hayashi $\mathrm{C}$, et al. Multiple molecular interactions implicate the connectin/titin N2A region as a mod- ulating scaffold for p94/calpain 3 activity in skeletal muscle. J Biol Chem. 2008;283(21):14801-14814.

18. Witt CC, et al. Induction and myofibrillar targeting of CARP, and suppression of the $\mathrm{Nkx} 2.5$ pathway in the MDM mouse with impaired titin-based signaling. J Mol Biol. 2004;336(1):145-154.

19. Ojima K, et al. Myogenic stage, sarcomere length, and protease activity modulate localization of muscle-specific calpain. J Biol Chem. 2007; 282(19):14493-14504.

20. Murphy RM, Goodman CA, McKenna MJ, Bennie J, Leikis M, Lamb GD. Calpain-3 is autolyzed and hence activated in human skeletal muscle $24 \mathrm{~h}$ following a single bout of eccentric exercise. J Appl Physiol. 2007;103(3):926-931.

21. McNeil PL, Khakee R. Disruptions of muscle fiber plasma membranes. Role in exercise-induced damage. Am J Pathol. 1992;140(5):1097-1109.

22. Wang J, et al. Dynamics of Z-band based proteins in developing skeletal muscle cells. Cell Motil Cytoskeleton. 2005;61(1):34-48.

23. Tothova J, et al. NFATc1 nucleocytoplasmic shuttling is controlled by nerve activity in skeletal muscle. J Cell Sci. 2006;119(pt 8):1604-1611.

24. Puchner EM, et al. Mechanoenzymatics of titin kinase. Proc Natl Acad Sci U S A. 2008;105(36):13385-13390.

25 . Ono Y, et al. Suppressed disassembly of autolyzing p94/CAPN3 by N2A connectin/titin in a genetic reporter system. J Biol Chem. 2006; 281(27):18519-18531.

26. Hentzen ER, et al. Stress-dependent and -independent expression of the myogenic regulatory factors and the MARP genes after eccentric contractions in rats. J Physiol. 2006;570(pt 1):157-167.

27. Lehti M, Kivela R, Komi P, Komulainen J, Kainulainen $\mathrm{H}$, Kyrolainen $\mathrm{H}$. Effects of fatiguing jumping exercise on mRNA expression of titin-complex proteins and calpains. J Appl Physiol. 2009;106(4):1419-1424.

28. Kojic S, et al. The Ankrd2 protein, a link between the sarcomere and the nucleus in skeletal muscle. JMol Biol. 2004;339(2):313-325.

29. Anderson LV, et al. Dysferlin is a plasma membrane protein and is expressed early in human development. Hum Mol Genet. 1999;8(5):855-861.

30. Ampong BN, Imamura M, Matsumiya T, Yoshida $\mathrm{M}$, Takeda S. Intracellular localization of dysferlin and its association with the dihydropyridine receptor. Acta Myol. 2005;24(2):134-144.

31. Stockholm D, Bartoli M, Sillon G, Bourg N, Davoust J, Richard I. Imaging calpain protease activity by multiphoton FRET in living mice. J Mol Biol. 2005;346(1):215-222.

32. Diaz BG, Moldoveanu T, Kuiper MJ, Campbell RL, Davies PL. Insertion sequence 1 of muscle-specific calpain, p94, acts as an internal propeptide. J Biol
Chem. 2004;279(26):27656-27666.

33. Rey MA, Davies PL. The protease core of the muscle-specific calpain, p94, undergoes $\mathrm{Ca}^{2+}$ dependent intramolecular autolysis. FEBS Lett. 2002;532(3):401-406.

34. Miller MK, et al. The muscle ankyrin repeat proteins: CARP, ankrd2/Arpp and DARP as a family of titin filament-based stress response molecules. JMol Biol. 2003;333(5):951-964.

35. Pallavicini A, et al. Characterization of human skeletal muscle Ankrd2. Biochem Biophys Res Commun. 2001;285(2):378-386.

36. Bean C, Facchinello N, Faulkner G, Lanfranchi G. The effects of Ankrd2 alteration indicate its involvement in cell cycle regulation during muscle differentiation. Biochim Biophys Acta. 2008; 1783(6):1023-1035.

37. Keira Y, Noguchi S, Minami N, Hayashi YK, Nishino I. Localization of calpain 3 in human skeletal muscle and its alteration in limb-girdle muscular dystrophy 2A muscle. J Biochem. 2003;133(5):659-664.

38. Shin JH, Kim HS, Lee CH, Kim CM, Park KH, Kim DS. Mutations of CAPN3 in Korean patients with limb-girdle muscular dystrophy. J Korean Med Sci. 2007;22(3):463-469.

39. Sanger JM, Mittal B, Pochapin MB, Sanger JW. Myofibrillogenesis in living cells microinjected with fluorescently labeled alpha-actinin. J Cell Biol. 1986;102(6):2053-2066

40. Takekura H, Fujinami N, Nishizawa T, Ogasawara H, Kasuga N. Eccentric exercise-induced morphological changes in the membrane systems involved in excitation-contraction coupling in rat skeletal muscle. J Physiol. 2001;533(pt 2):571-583.

41. Matsuda R, Nishikawa A, Tanaka H. Visualization of dystrophic muscle fibers in $\mathrm{mdx}$ mouse by vital staining with Evans blue: evidence of apoptosis in dystrophin-deficient muscle. J Biochem. 1995;118(5):959-964.

42. Ojima K, et al. Mac-1(low) early myeloid cells in the bone marrow-derived SP fraction migrate into injured skeletal muscle and participate in muscle regeneration. Biochem Biophys Res Commun. 2004; 321(4):1050-1061.

43. Sorimachi H, et al. Muscle-specific calpain, p94, is degraded by autolysis immediately after translation, resulting in disappearance from muscle. J Biol Chem. 1993;268(14):10593-10605.

44. Ono Y, et al. Comprehensive survey of p94/calpain 3 substrates by comparative proteomics-possible regulation of protein synthesis by $\mathrm{p} 94$. Biotechnol J. 2007;2(5):565-576.

45. Ihaka R, Gentleman R. R: a language for data analysis and graphics. J Comp Graph Stat. 1996;(3):299-314. 